\title{
SeaFish: A Game for Collaborative and Visual Image Annotation and Interlinking
}

\author{
Stefan Thaler ${ }^{1}$, Katharina Siorpaes ${ }^{1}$, David Mear ${ }^{3}$, \\ Elena Simperl ${ }^{1,2}$, and Carl Goodman ${ }^{3}$ \\ 1 University of Innsbruck, STI-Innsbruck, Innsbruck, Austria \\ firstname.secondname@sti2.at \\ ${ }^{2}$ Karlsruhe Institute of Technology, AIFB, Karlsruhe, Germany \\ firstname.secondname@kit.edu \\ 3 Pepper's Ghost Productions, London, UK \\ firstname.secondname@peppersghost.com
}

\begin{abstract}
Many tasks in semantic content creation, from building and aligning vocabularies to annotation or data interlinking, still require human intervention. Even though automatic methods addressing the aforementioned challenges have reached a certain level of maturity, user input is still required at many ends of these processes. The idea of human computation is to rely on the human user for problems that are impossible to solve for computers. However, users need clear incentives in order to dedicate their time and manual labor to tasks. The OntoGame series uses games to hide abstract tasks behind entertaining user interfaces and gaming experiences in order to collect knowledge. SeaFish is a game for collaborative image annotation and interlinking without text. In this latest release of the OntoGame series, players have to select images that are related to a concept that is represented by an image (from DBpedia) from a collection of images (produced by querying flickr ${ }^{T M}$ wrappr with the respective concept). The data collected by SeaFish is published as Linked Data on the Web. In this paper we outline the SeaFish game and demo.
\end{abstract}

Keywords: Games for semantic content creation, Image annotation, Linked Open Data, multimedia interlinking.

\section{Motivation}

The automated interpretation and description of images is still a major challenge. CAPTCHAs are still a prime example for a task that is trivial for a human user but impossible for a computer [6], allowing the distinction between a human user and a machine. The paradigm of human computation is also the foundation for "games with a purpose" [4] which aim to exploit human intelligence for solving computationally difficult tasks by masquerading them as games. Thereby, an abstract (knowledge acquisition or curation) task is not only hidden behind an attractive, easy-to-understand user interface but users are also given incentives to dedicate their time to solving the task. Playing, competition, and social

G. Antoniou et al. (Eds.): ESWC 2011, Part II, LNCS 6644, pp. 466-470, 2011.

(C) Springer-Verlag Berlin Heidelberg 2011 
recognition are core motivational mechanisms of games. The OntoGame series uses casual games in order to acquire human input for several semantic content creation tasks, such as ontology building (e.g. the OntoPronto game), video annotation (e.g. the OntoTube game), or ontology alignment (e.g. the SpotTheLink game).

In this paper we present the SeaFish game and describe its demo as planned for ESWC 2011. SeaFish is the latest release of the OntoGame series. SeaFish is a game for collaborative image annotation and interlinking without text. Players have to select images that are related to a concept that is represented by an image (from DBpedia) from a collection of images (produced by querying flickr ${ }^{T M}$ wrappr 1 with the respective concept). Players get points based on their degree of consensus with the player community. The data collected by SeaFish is published as Linked Data on the Web.

SeaFish does not involve any reading for annotation. It solely relies on images. This means that the game can be played in a fast pace as reading takes more time than just looking at a visual representation of a concept. Moreover, the game is not constrained by language or reading limitations. The resulting data is published as Linked Data and thus, SeaFish implements game-based multimedia interlinking as described by Buerger and colleagues [2].

Related work. We sketch related work focusing on games for the creation of image annotations. A comprehensive list of games for knowledge acquisition is available at the INSEMTIVES homepag£2. Luis von Ahn introduced the ESP game[7] aka Google's Image Labeler for annotating images. Additioinally, he created other serious games that are published at the GWAP homepage 3 . PopVided 4 aims to label videos and Waisda5 allows players to tag live TV shows. Magic Bullet 9] evaluates the robustness of Captchas, TagCaptcha [3] produces image annotations as side product of the CAPTCHA process. Finally, Picture This 6 is another game for annotating images, however, unlike the ESP game users don't create tags but have to decide on the best matching tag.

\section{Collaborative and Game-Based Image Annotation}

SeaFish SeaFish is a single player game where players have to fish related images that are floating around. Users can play from the OntoGame website 7 as either registered user or as anonymous guest. Players may also login via Facebook by visiting the OntoGame Facebook page 8 . After they clicked the SeaFish! button

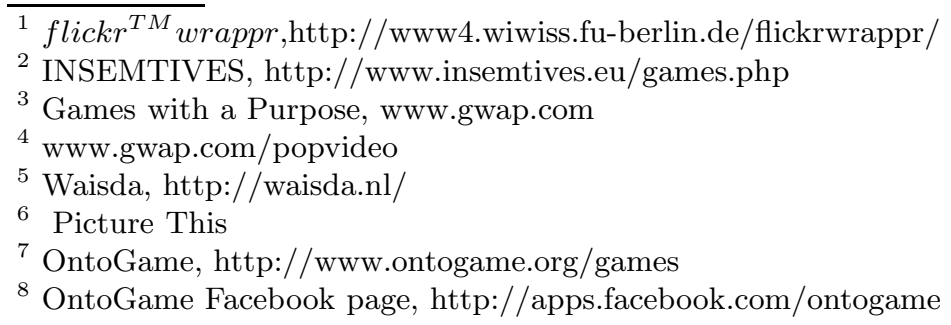




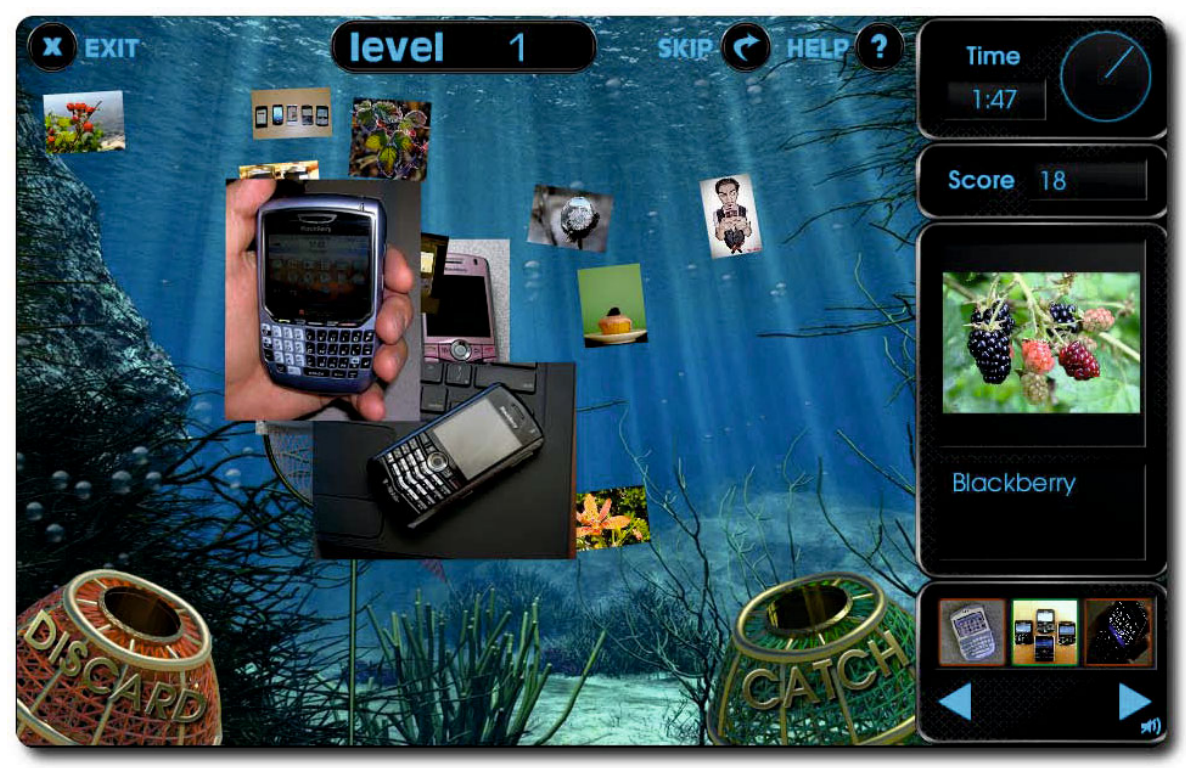

Fig. 1. SeaFish - in game screen

the game starts. From there players may either get playing instructions or start a game. Each game round is about a concept taken from DBpedia that is represented by an image. Players see this image on the right hand side. Additionally, players see the result of an search for the concept on flickr ${ }^{T M}$ wrappr, which are floating through the main screen (see Figure 1). Players have 2 minutes to mark those images as either related to the concept or unrelated to the concept. They can do so by catching the images with the fishing rod and dragging them either to the green basket (related) or the red basket (not related).

Players always obtain a small reward for catching an image. Generally, players get more points depending on whether their decision is consensual with decisions of the majority of other players. Additionally, the reward a player gets always depends on their reliability as well as their level. When an image is caught a new image appears in order to have less than ten images floating on the screen at the same time. The game stops when all images have been fished or the time is up. After each game round (see Figure 2) players can compare their answers with the communitys answers as well as view statistics on accuracy and time.

To give an example of Seafish: in a game round the player is shown an image of Blackberry (see Figure 1) on the right hand side (retrieved from DBpedia). As a result of the query Blackberry on flickr ${ }^{T M}$ wrappr, pictures are floating around on the main screen. The task of the player is to select those image that are related to the concept Blackberry(the berry) and those that are not by putting images in the "Discard" or "Catch" baskets on the bottom of the screen. 


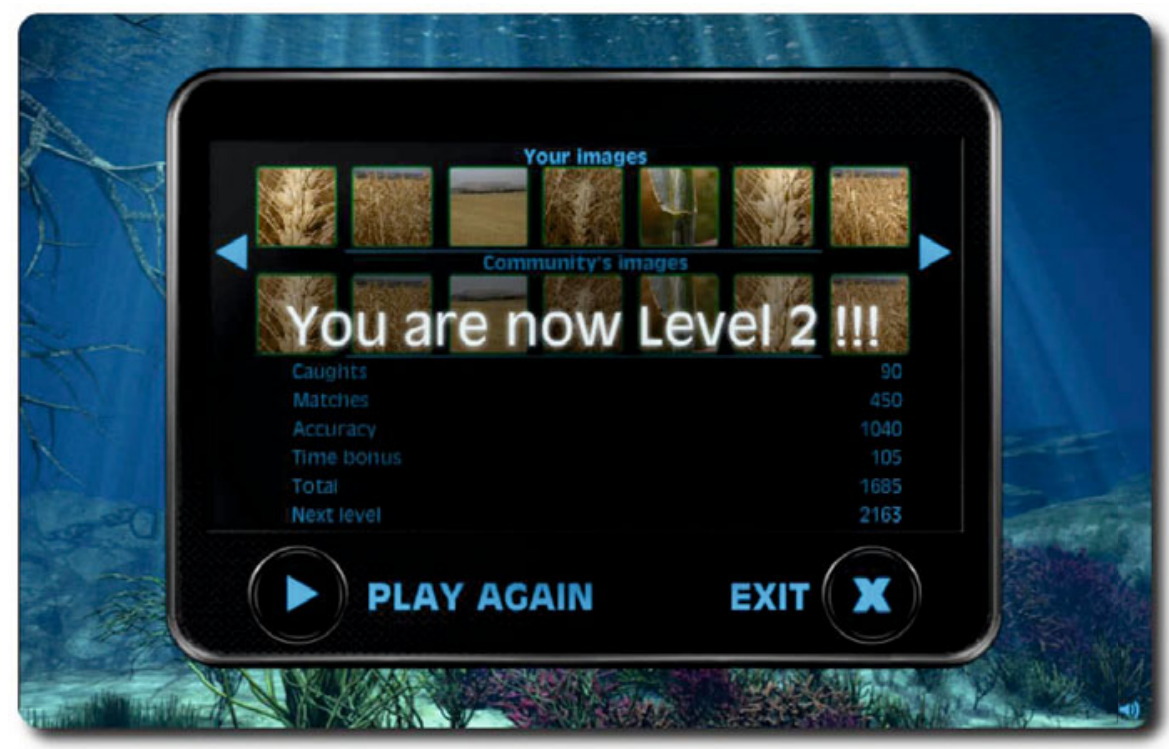

Fig. 2. SeaFish - comparison screen

Data export. SeaFish exports annotations as RDF triples. In our first scenario we create annotations of the form $<$ image $><$ http://xmlns.com/foaf/spec/depiction $><$ concept $>$ as well as the inverse property. Our concepts are taken from DBpedia plus follow the four Linked Open Data principles 11. This means that our data may be used to contribute to the Linked Open Data cloud without being modified.

Our annotations are generated from stored answers in the following manner: let $s_{i}$ be an answer that states an image is related to a concept, $i$ a number greater than six, $r_{i}$ the reliability of the player giving the answer at the time of the answer and $n$ the number of total answers stored about an image. A player's reliability is a measurement of how accurate this player played in previous games.

\section{Discussion and Outlook}

Currently, three months after the initial release of SeaFish we have collected feedback, implemented the proposed changes and re-released the game. A still open issue is the lack of returning player. To counter this we have integrated leveling mechanisms. We have integrated it on Facebook to profit from the social network effect. We are currently evaluating the outcome of these measures. Besides, we also evaluate the effect of tweaking the rewarding function on the quality of the generated annotations. 
We also identified a possible extension of SeaFish: Ookabod9 is an API that links pictures to concepts from different Linked Open Data sources and makes them searchable. SeaFish could on the one hand be used to verify the data provided on Ookaboo. On the other hand it could also be used to provide new image tags.

Another possibility to attract more players is a planned feature to allow users to add their own category of concepts to play in the games.

\section{Conclusion}

At ESWC 2011 the audience of the demo players will be able to play the games of the OntoGame series (including Seafish) and can see how and which data is generated. In this paper, we have described the SeaFish game for collaborative image annotation. We are currently collecting massive user input in order to thoroughly evaluate the game by assessing the quality of generated data and the user experience.

\section{Acknowledgments}

The work presented has been funded by the FP7 project INSEMTIVES under EU Objective 4.3 (grant number FP7-231181).

\section{References}

1. Berners-Lee, T.: Linked data - design issues (2006)

2. Hausenblas, M., Troncy, R., Raimond, Y., Bürger, T.: Interlinking multimedia: How to apply linked data principles to multimedia fragments. In: Linked Data on the Web Workshop, LDOW 2009 (2009)

3. Morrison, D., Marchand-Maillet, S., Bruno, E.: Tagcaptcha: annotating images with captchas. In: Proceedings of the ACM SIGKDD Workshop on Human Computation, HCOMP 2009, pp. 44-45. ACM, New York (2009)

4. Von Ahn, L.: Games with a purpose. IEEE Computer 29(6), 92-94 (2006)

5. Von Ahn, L.: Peekaboom: A Game for Locating Objects in Images (2006)

6. Von Ahn, L., Blum, M., Hopper, N.J., Langford, J.: Captcha: Using hard ai problems for security (2003)

7. Ahn, L.v., Dabbish, L.: Labeling images with a computer game. In: CHI, pp. 319-326 (2004)

8. von Ahn, L., Ginosar, S., Kedia, M., Blum, M.: Improving Image Search with PHETCH. In: IEEE International Conference on Acoustics, Speech and Signal Processing, ICASSP 2007, vol. 4, pp. IV-1209 -IV-1212 (2007)

9. Yan, J., Yu, S.-Y.: Magic bullet: a dual-purpose computer game. In: Proceedings of the ACM SIGKDD Workshop on Human Computation, HCOMP 2009, pp. 32-33. ACM, New York (2009)

${ }^{9}$ Ookaboo, http://ookaboo.com/ 